Fortpflanzungsgeschwindigkeit der erdmagnetischen Störungen vom 12. u. 15. Nov. 1905 und ihr Vergleich mit dem Fortschreiten der gleichzeitigen Sonnenflecken.

Die erdmagnetischen Ungewitter, die sich gelegentlich der Wiederkehr der im Oktober 1905 durch eine ungewöhnlich große Gruppe von Sonnenflecken signalisierten Steigerung der Sonnentätigkeit im November 1905 ereigneten, hatten den besonderen Vorzug, für europäische Stationen mehrere Stunden nach Sonnenuntergang zu entfallen. Dem entsprach eine seltene Schärfe ihrer Ausprägung in Kurven. Die eigenartig einfachen Zacken der Deklinationsstörung sind von Mr. Chree an den im Kew Observatorium, von M. Moureaux besonders an den zu Val Joyeux registrierten Kurven bereits in Nature und in den Comptes rendus \& der Pariser Akademie beschrieben. Sie finden sich an den gleichen Abenden des 12 . und I5. November 1905 auch auf den Deklinationskurven des Potsdamer und, jedenfalls am I 2., auf derjenigen des Pawlowsker Observatoriums wieder, die dem Unterzeich. neten mit höchst dankenswerter Liberalität von den Vorständen Herren A. Schmidt und W. Dubinsky zur Verfügung gestellt wurden. Sie ließen zum ersten Male in zweifelloser Weise eine zeitliche Folge der erdmagnetischen Störungen von Station zu Station erkennen. Diese gestattete an den Störungen die Richtung ihres Weges und die Geschwindigkeit ihrer Wanderung von Ort zu Ort festzustellen.

Die an den Deklinationskurven der drei Stationen Pawlowsk, Potsdam, Kew bisher gewonnenen Ergebnisse sind folgende:

I. Die Richtung war überall eine ausgeprägt westliche.

2. Die Geschwindigkeit war an beiden Abenden die gleiche, da der Weg von Potsdam bis Kew beidemale in etwa $81 / 2$ Zeitminuten zurückgelegt wurde.

Großflotbek, 1906 Februar 9.
3. Sie ergab sich in diesem Intervall zu 2119 sem., reduziert auf den für die Nordhemisphäre der Erde ungefähr mittleren und zugleich Kew und Potsdam sehr nahe gelegenen Parallel von $50^{\circ}$ nördl. Breite.

4. Die Geschwindigkeit von Meridian Pawlowsk nach Meridian Potsdam, reduziert auf denselben Parallel von $50^{\circ}$ nördl. Breite, ergab am I2. November einen dem unter 3. angegebenen sehr nahestehenden Wert von $1966 \mathrm{sem}$. Für den 15. November war sie deshalb nicht mit Sicherheit festzustellen, weil bei Pawlowsk eine genügend scharf ausgeprägte Zacke der Kurve fehlte.

5. Die tellurischen Geschwindigkeitswerte, 3. und 4., gehören derselben Größenordnung an wie der Geschwindigkeitswert eines solaren Vorgangs, dessen Zusammen. hang mit erdmagnetischen Störungen schon aus anderen Gründen sehr wahrscheinlich gemacht ist. Gemeint ist die gleichzeitige örtliche Steigerung der Sonnentätigkeit, die optisch signalisiert wird durch das Auftreten von Sonnenflecken. Für die Epoche im November I $905 \mathrm{kam}$ eine Zone von Sonnenflecken in Betracht, in der von M. Moureaux und unabhängig, doch in einer etwas späteren Veröffentlichung vom Unterzeichneten die Wiederkehr der großen Gruppe vom 20. Oktober 1905 erkannt wurde. In Übereinstimmung mit Herrn G. Spörers älteren Ergebnissen für die gleiche heliographische Breite, betrug ihre Bewegung, ebenfalls nach westlicher Richtung, rund 14 Längengrade der Sonne in 24 Stunden. Die daraus berechnete Sekundengeschwindigkeit beträgt I 849 sem., ein Wert, der hinter 3. nur um 13 , hinter 4 . sogar nur um 6 Prozent zurücksteht.

Wilhelm Krebs.

\title{
Beobachtungen auf der Privatsternwarte Jena im Jahre 1905.
}

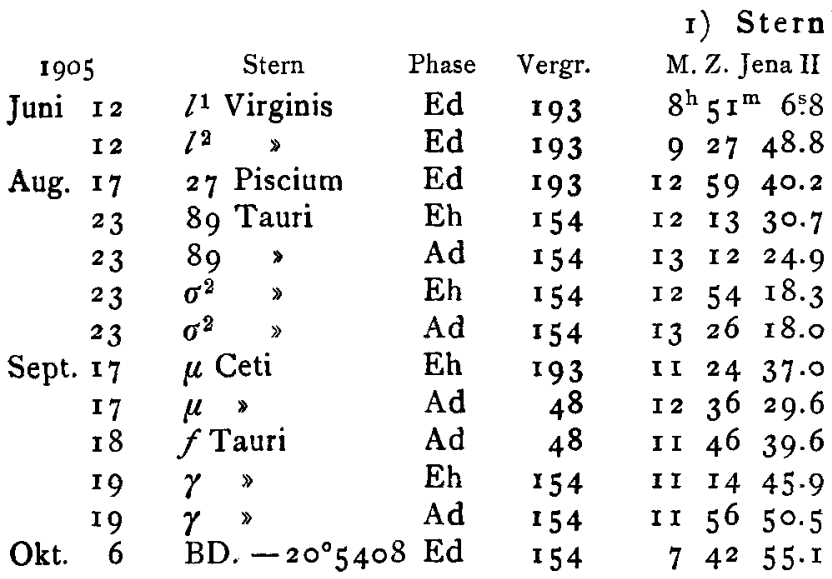

Sämtliche Beobachtungen am 6-Zöller. Zeitbestimmung stets im unmittelbaren Anschluß an die Beobachtungen. Koeffizienten sind Logarithmen.

Juni I 2. $l^{1}$ Virginis etwas unsicher; vielleicht $\mathbf{I}_{2}^{\mathbf{s}} \mathbf{z u}$ spät? $l^{2}$ Virginis vielleicht 0.4 verspätet. - Aug. 23. 89 Tauri Eh sehr unruhige Luft, Stern schwer zu sehen. $\sigma^{2}$ Tauri Eh

$$
\begin{aligned}
& \text { hieraus } \\
& 0=+7.5+0.035128 \Delta(a-\alpha)-0.298707 \Delta(d-\delta) \\
& -17.4+0.294326 \text { + } \quad+8.830480 \text { \% } \\
& -16.5+0.264844 \text { } \gg+9.8844 \mathrm{r} 5 \text { 》 } \\
& -22.4+0.273762 \text { + }+9.277779 \text { 》 } \\
& +2.6-9.874198 \text { 》 }-9.900246 \text { 》 } \\
& -20.2+0.35444^{8} \gg-0.360370 \% \\
& -14.2-0.114281,-0.627248 \text {, } \\
& -18.3+0.231576 \geqslant+0.122373 \\
& -10.9-0.300253,-9.468702 \text {, } \\
& -15.8+0.295624 \gg+9.333454 \text { 》 } \\
& -19.3+0.140921 \%+0.504347 \% \\
& -10.5-0.348485 \% \quad+0.177864 \text {, } \\
& -1.4+9.503749 \geqslant-0.262237
\end{aligned}
$$

etwas unruhig. - Okt. 6. Trotz leichter Wolkenschleier ziemlich gut. Die Angabe des Beobachtungsbuches: Ed $7^{\mathrm{h}} 43^{\mathrm{m}} 55^{\mathrm{s}} \mathrm{r}$ wohl $u m r^{m}$ irrig. Der mittlere Ort des Sterns wurde angenommen:

$$
\text { r } 905 \text { I } 9^{\mathrm{h}} 3^{\mathrm{m}} \times 6^{\mathrm{s}} \cdot 79-\mathrm{r} 9^{\circ} 57^{\prime} 54^{\prime \prime} \circ \mathrm{AOe}_{2} \text { I9 } 127 .
$$

